\title{
AÇÕES DO ENFERMEIRO DA ESTRATÉGIA SAÚDE DA FAMÍLIA NA PROMOÇÃO DO ENVELHECIMENTO SAUDÁVEL
}

\section{Nurse's shares of Family Health Strategy in healthy aging promotion}

Neiva Junkes Hoepers ${ }^{1}$, Lusiane Mendes Nascimento ${ }^{1}$, Suelen Biz Urbano ${ }^{1}$, Diogo Dominguini ${ }^{1}$, Susane Raquel Périco Pavei ${ }^{1}$, Maria Teresa Brasil Zanini ${ }^{1}$, Paulo Rogério Hansen ${ }^{1}$

1Departamento de Enfermagem - Unidade Acadêmica de Ciências da Saúde UNASAU, Universidade do Extremo Sul Catarinense - UNESC, Criciúma, SC, Brasil.

\section{Endereço para correspondência:}

Neiva Junkes Hoepers

Departamento de Enfermagem - Unidade Acadêmica da Saúde - UNASAU - UNESC Av. Universitária, 1105. Criciúma - SC - Bairro Universitário. CEP - 88806-000.

Email: neivajun@unesc.net 


\title{
Resumo
}

O presente trabalho teve como sujeitos da pesquisa os enfermeiros, trabalhadores das Estratégias Saúde da Família de um Município do Sul Catarinense. Como protagonistas apresentamos os idosos, população esta, crescente, necessitando de cuidados e tratamentos específicos, buscando identificar ações do enfermeiro na Estratégia Saúde da Família quanto à promoção de um envelhecimento saudável com qualidade de vida e autonomia. A questão do envelhecimento demográfico, relacionado ao aumento da expectativa de vida e uma diminuição das taxas de fecundidade, vem apresentando desafios ao setor saúde, despertando provocações aos enfermeiros das Estratégias Saúde da Família e aos demais trabalhadores da saúde, pois trabalham diretamente no cotidiano com esta demanda, necessitando desenvolver ações que promovam um envelhecimento saudável. O objetivo deste estudo foi de conhecer as ações do enfermeiro das Estratégias Saúde da Família na promoção do envelhecimento saudável. Para isso foram realizadas entrevistas semiestruturadas, abrangendo todas (cinco) as equipes de Estratégia Saúde da Família do município do estudo, totalizando cinco enfermeiros, no período entre março e maio de 2015. Evidenciou-se a falta de ações específicas ao envelhecimento saudável no trabalho cotidiano dos enfermeiros nas Estratégia Saúde da Família, pela falta de apoio ou incentivo para Atenção Primária de Saúde, com ações de promoção/prevenção contribuindo com um envelhecimento ativo, humanizado e com qualidade de vida.

Palavras-chave: Saúde do Idoso; Promoção da Saúde; Enfermeiros; Envelhecimento da População.

\begin{abstract}
Abstratc
This work had as research subjects nurses, workers of the Health Strategy of the Family of a Far South Santa Catarina Municipality. As protagonists present the elderly population is, growing, requiring specific care and treatment in order to identify the nurse's actions in the Family Health Strategy as the promotion of healthy aging with quality of life and autonomy. The question of aging, related to increased life expectancy and reduced fertility rates, has presented challenges to the health sector, arousing provocations to nurses of the Family Health Strategy and other health workers,
\end{abstract}


Artigo Original

Atenção à Saúde

because they work directly in daily with this demand, need to develop actions to promote healthy aging. The aim of this study was to know the actions of the nurse of the Family Health Strategies in promoting healthy aging. For this semi-structured interviews were conducted, covering all (five) Health Strategy teams study the county family, totaling five 05 nurses in the period between March and May 2015. It was evidenced the lack of specific actions to healthy aging in daily work of nurses in the Family Health Strategy, the lack of support or incentive for primary health care, with actions of promotion / prevention contributing to active aging, humanized and quality of life.

Keywords: Elderly Health; Health Promotion; Nurses; Aging Population.

\section{INTRODUÇÃO}

No Brasil, ao longo dos anos, percebe-se que a população idosa vem aumentando, acompanhando uma tendência mundial ${ }^{1}$. Estudos apontam que em 2025 o Brasil deverá possuir a sexta maior população idosa do mundo, chegando a um número de 32 milhões de pessoas com idade acima de 60 anos $^{2,3}$.

O envelhecimento saudável aparece no contexto abordado por vários autores, entre eles Lenardt et al. ${ }^{4}$ onde considera que a velhice tem sido assunto de vários estudos empregados no conjunto de ciências, técnicas e saberes, o qual observa-se uma estreita relação entre hábitos de vida saudável, disciplina alimentar, atividade física regular e/ou prolongada, obter e manter hábitos saudáveis, ou seja, despertar o bom senso para ter um envelhecimento saudável ou pelo menos promover esse envelhecimento ${ }^{4}$.

A Política Nacional do Idoso vem se estabelecendo ao longo das últimas décadas, com o intuito de buscar a garantia dos direitos às pessoas, levando em consideração o aumento da expectativa de vida da população, ao passo em que novas estatísticas vêm indicando o crescimento da população nesta faixa etária ${ }^{5}$. Sendo assim, o Ministério da Saúde ${ }^{6}$ propõe uma atenção contínua e eficaz para a saúde e o bem-estar da população idosa, requerendo diferentes níveis de intervenção dos serviços de saúde, condizentes às distintas fases da enfermidade e ao grau de 


\section{Artigo Original}

\section{Atenção à Saúde}

incapacidades, que devam basear-se em última instância em uma atenção integral, adequada, de qualidade, humanizada e oportuna.

O cuidado ao idoso, na área da enfermagem, foi por muitos anos relegado a segundo plano na assistência. Porém hoje, com a implantação da Estratégia de Saúde da Família (ESF), vem mudando o contexto histórico e modificando a forma de assistir essa parcela da população, desenvolvendo ações de proteção e promoção da saúde, assim como a reabilitação e prevenção de agravos à mesma ${ }^{7,8}$. Com a Política Nacional de Humanização (PNH) e o estatuto do idoso, o Ministério da Saúde propõe estimular e incentivar a valorização dos profissionais de saúde que fazem parte desse processo ${ }^{6}$. O enfermeiro, como protagonista do cuidado, tem um papel essencial nesse processo, pois precisa saber identificar no contexto do envelhecimento o que faz parte do envelhecer "normal", suas limitações fisiológicas gradativas e as características patológicas que podem instalar-se nesse processo, identificando precocemente as patologias e trabalhando de forma participativa e efetiva, gerando grande desafio ao profissional enfermeiro e à Saúde Pública ${ }^{2,9}$.

A Atenção Básica $(\mathrm{AB})$ deve ser o contato preferencial dos usuários, a principal porta de entrada e centro de comunicação com toda a Rede de Atenção à Saúde. Por isso, é fundamental que ela se oriente pelos princípios da universalidade, da acessibilidade, do vínculo, da continuidade do cuidado, da integralidade da atenção, da responsabilização, da humanização, da equipe e da participação social ${ }^{5}$. Muitas ações são desenvolvidas pela equipe da Estratégia Saúde da Família (ESF), entre estas se deve promover o acolhimento digno e humanizado e realizar atividades de educação em saúde que visem à promoção da saúde, bem estar físico, mental e social do idoso, como também promover ações para prevenir o isolamento social, a doença mental e a violência neste grupo específico, entre outras que podem ser agregadas à equipe de saúde ${ }^{10}$.

Diante dessas reflexões tem-se como problema de pesquisa: Quais as ações do enfermeiro da Estratégia Saúde da Família (ESF) na promoção do envelhecimento saudável, em um município do Sul de Santa Catarina? Foi elencado como objetivo deste estudo: conhecer as ações do enfermeiro das Estratégias Saúde da Família na promoção do envelhecimento saudável.

Acreditamos que, com os resultados obtidos, esses profissionais sejam capazes de qualificar sua assistência e oferecer a esta faixa etária um atendimento 
Artigo Original

Atenção à Saúde

singular e baseado em suas reais necessidades de saúde, ponderando as especificidades do processo de envelhecimento.

\section{METODOLOGIA}

A abordagem da pesquisa foi qualitativa do tipo descritiva, exploratória e de campo com a aplicação de entrevista semiestruturada ${ }^{11}$. O estudo foi desenvolvido nas Estratégias de Saúde da Família de um Município do Sul de Santa Catarina. O município possui cinco equipes de ESF com abrangência total de seu território.

Foram realizadas entrevistas semiestruturadas com todos os enfermeiros das cinco ESF, que aceitaram participar da pesquisa e assinaram o Termo de Consentimento Livre e Esclarecido (TCLE), conforme a resolução 466/12, totalizando 05 enfermeiros.

Primeiramente foi solicitada a autorização para a realização da pesquisa e reconhecimento do cenário de aplicação da mesma. Após foi apresentado o projeto de pesquisa e agendadas as entrevistas aos sujeitos da pesquisa. Com o local, data e horário marcado foram aplicadas as entrevistas, com a manifestação do aceite através da assinatura do Termo de Consentimento Livre e Esclarecido (TCLE). A coleta dos dados aconteceu nos meses de março a maio de 2015, após a aprovação do projeto pelo comitê de ética da Universidade do estudo, sob o número 972.316 de 2015.

Foi caracterizado o perfil dos Enfermeiros, sendo denominadas E1, E2, E3, E4 e E5 para as falas, assim, preservando o sigilo e questões éticas dos entrevistados.

A análise e interpretação dos dados foram através da ordenação, classificação e análise final dos dados, de acordo com a categorização apresentada por Minayo ${ }^{3}$.

\section{RESULTADOS E DISCUSSÃO}

Realizou-se a categorização dos dados, organizando-se as unidades de registro a partir de suas afinidades temáticas: Idosos acamados e a assistência prestada; As dificuldades ao prestar assistência aos idosos; Educação em saúde prestada pela Estratégia Saúde da Família; Desenvolvimento de ações que são 
Artigo Original

Atenção à Saúde

preconizadas pelo Ministério da Saúde ao idoso; Planejamento e assistência da saúde do idoso na Estratégia Saúde da Família. E na interpretação buscou-se a compreensão e interpretação dos dados à luz do referencial ${ }^{12}$.

\section{Idosos Acamados e a Assistência Prestada}

$\mathrm{Na}$ análise das ações voltadas ao idoso, percebeu-se que $\mathrm{o}$ atendimento domiciliar é uma das principais ações desenvolvidas pela equipe de saúde ao paciente acamado. E nos atendimentos domiciliares mencionados como "visitas domiciliares", é onde se desenvolve a assistência conforme a necessidade singular de cada idoso. Este fato é percebido nos relatos:

E1: Realiza assistência através de visitas domiciliares médicas, enfermagem.

E2: Possuem dois acamados no momento, prestamos visita domiciliar (médico, enfermeira, técnica de enfermagem e ACS), e no NASF também dependendo da necessidade do acamado estamos sempre à disposição para prestar a assistência, por exemplo: orientação sobre medicação, administração de medicamento injetável, controle de sinais vitais, fisioterapia, nutrição e psicóloga, prevenção de Ulcera Por Pressão (UPP), orientação sobre os direitos dos idosos.

E3: Esta unidade não possui idoso acamado.

E4: Possuem 17 idosos acamados e prestam assistência realizando as visitas domiciliares pela ESF, com a equipe de enfermagem e médico. Como também, curativo domiciliar, apoio NASF.

E5: Presta assistência através de visita domiciliar do médico, enfermeira, técnica de enfermagem e NASF.

Para Flesch ${ }^{13}$, a Visita Domiciliar no setor público justifica-se pelo grau de humanização que essa atenção traz para o atendimento ao usuário/família, pela possibilidade de desospitalização, com liberação de leitos para doentes, que realmente necessitam destes, como também pela redução de complicações 
Artigo Original

Atenção à Saúde

decorrentes de longas internações hospitalares com redução dos custos envolvidos em todo o processo de hospitalização ${ }^{13}$.

Por outro lado, vindo ao encontro a essa avaliação, ressalta-se também a sobrecarga e as dificuldades de trabalho das equipes, por ser a Atenção Primária em Saúde (APS) a ordenadora do cuidado e ressaltando as altas demandas e a falta de investimento de recursos humanos, como também o déficit de recursos materiais e a carência de apoio das secretarias de saúde em relação aos meios para prestar atendimentos ${ }^{7,8,14}$.

\section{As Dificuldades ao Prestar Assistência aos Idosos}

No entendimento dos enfermeiros uma das maiores dificuldades é a resistência ao cuidado, vindo ao encontro com o que alguns autores colocam sobre a caracterização da velhice, como sendo a fase final do ciclo da vida, trazendo com ela algumas manifestações físicas, psicológicas, sociais e debilidades. Dentre as quais podemos destacar com mais intensidade a diminuição da capacidade funcional, trabalho e resistência, aparecendo também a solidão, calvície, perda de papéis sociais e culturais importantes, prejuízos psicológicos, motores e afetivos ${ }^{8}$.

Desta forma, podemos destacar algumas falas, das dificuldades relatadas pelos enfermeiros, que também podem ser dificuldades encontradas nesta fase da vida das pessoas:

E1: Tem alguns que são um pouco mais resistentes, como por exemplo: às vezes o cuidador relata que o idoso necessita utilizar fraldas para dormir, mas o idoso acaba resistindo à utilização, o que acaba urinando a noite na cama e muitas vezes ficando molhado até o dia seguinte. Em outros casos os idosos querem ser muito independentes, mas já não conseguem ler os medicamentos, acabam tomando a medicação de forma incorreta, pois muitos se baseiam pela cor do comprimido, etc.

E2: Dificuldade dependendo do problema do paciente em aceitar o tratamento.

E3: Dificuldade de aceitar que tem que consultar, resistência no uso de medicação, orientar como deve tomar a medicação, falta 
de um familiar que acompanhe o idoso.

E4: O fato de não estar bem preparada para essa assistência, devido a falta de cursos e capacitações, bem como a falta de órgãos públicos para prestar suporte e apoio às equipes.

E5: Muitos possuem dificuldade cognitiva, na fala, assim dificulta o entendimento dos profissionais, esta é a maior dificuldade.

Entre as dificuldades na fase idosa, relatadas anteriormente, outros agravantes podem agregar a essa pessoa, para apresentar a resistência a um cuidado, como as mudanças tanto físicas, psíquicas como as fisiológicas, que vão se agravando com os anos de vida. Podendo ser enumeradas muitas outras como: papilas gustativas diminuem fazendo com que a pessoa sinta menos gosto pelos alimentos, a ausência dos dentes ou a utilização de próteses dentárias mal adaptadas dificulta na mastigação piorando em sua alimentação, há também alteração no movimento do esôfago fazendo com que a pessoa se engasgue com maior facilidade, a produção do ácido clorídrico também se mantém diminuída dificultando a digestão e absorção dos alimentos, ou seja, todas essas alterações reagem na mente do idoso fazendo com que ele fique chateado facilmente, mais teimoso, sensível às palavras, entre outras reações, que podem interferir em querer ajuda ${ }^{15}$.

Outro estudo mostra que a condição de vida influencia muito no processo do envelhecimento, pois quando existe um ambiente tranquilo, sem estresse, alimentação saudável, sono e repouso, estilo de vida adequado, aumenta a qualidade de vida de uma pessoa. Sendo assim, o envelhecimento é resultado das interações de fatores genéticos, ambientais e estilo de vida ${ }^{4}$.

\section{Educação em Saúde Prestada pela Estratégia Saúde da Família (ESF)}

No que se refere à educação em saúde prestada pela ESF, a promoção da saúde tenta resgatar um dos principais objetivos da saúde pública ou da saúde coletiva, tendo como meta não apenas afastar a doença do indivíduo, mas tomar como seu objetivo fundamental a atuação sobre os determinantes das doenças ${ }^{2}$.

Existe uma diferença conceitual em relação à prevenção e promoção de saúde, conceitos muitas vezes considerados sinônimos ou equivalentes ${ }^{5}$. A prevenção tem um caráter de intervenção diante de alguns agravos que podem 
Artigo Original

Atenção à Saúde

acometer indivíduos ou coletividades, utilizando determinadas tecnologias para evitar que tal condição mórbida diminua a ocorrência, ou ocorra de forma menos grave nos indivíduos ou nas coletividades. Já a promoção da saúde adquire um caráter permanente e visa à erradicação de ocorrência da mesma ${ }^{10}$.

Desta forma, podemos ver que os sujeitos da pesquisa realizam muito pouco atividades de educação em saúde. Foi descrito por uma pesquisada, que "às vezes" o Núcleos de Apoio à Saúde da Família (NASF), presta ajuda quando solicitado, conforme os relatos:

E1: Grupo de apoio não, pois na maioria das vezes é apenas uma pessoa da família que cuida do idoso, e geralmente essa pessoa dificilmente pode sair de casa, para cuidar de si.

E2; E3; E4: Não

E5: Não, apenas alguns profissionais, por exemplo, do NASF estão para prestar alguns tipos de cuidados e ajuda quando solicitados.

Portanto, a velhice deve ser compreendida em suas múltiplas dimensões e para isso precisamos da ampliação dos conhecimentos profissionais para além da área gerontogeriátrica, considerando as inter-relações, pois o idoso exige cuidado direcionado a ações complexas e interdisciplinares ${ }^{16}$.

Entre estas ações, podemos incluir a realização de visitas/atendimentos domiciliares regulares, garantia de consultas, tratamentos específicos para a fase da vida, medicamentos, exames laboratoriais e outros, de acordo com as necessidades individuais, orientações que envolvam o usuário e comprometam a família, garantir o atendimento nos diversos pontos de atenção, como o educador físico, o fisioterapeuta, o psicólogo, o nutricionista e o dentista ${ }^{17}$.

\section{Desenvolvimento de ações que são preconizadas pelo Ministério da Saúde (MS) ao idoso}

São medidas preconizadas pelo Ministério da Saúde como ações terapêuticas comuns na atenção básica, a necessidade de proporcionar ao usuário um momento para pensar/refletir, usando uma comunicação objetiva e clara realizando acolhimento com escuta qualificada, de modo a acolher o mesmo, respeitando as suas queixas 
Artigo Original

Atenção à Saúde

emocionais e o seu modo de vida ${ }^{14}$. Nas falas observa-se o relato da dificuldade de acesso dos pacientes, bem como pessoas capacitadas para atendimento voltado a pessoas idosas ou acamadas, até mesmo a estrutura física do local de atendimento.

E1: Não há.

E2: Dificuldade na adesão dos pacientes.

E3: Várias dificuldades: principal é o tempo, horário de atendimento pelo médico e pessoas capacitadas para acolher os idosos.

E4: Falta de espaço físico na ESF, pouco apoio de outros órgãos e profissionais, profissionais não capacitados para assistir ao idoso.

E5: Dificuldade de adesão dos mesmos, dificuldade de recursos financeiros, dificuldade e restrição na área física, dificuldade de tempo disponível pela enfermeira.

Acredita-se que a estratégia saúde da família abrange o cuidado ao idoso, e que este profissional já deve estar capacitado para este cuidado, não sendo ele um especialista, mas que tenha conhecimento e embasamento teórico e prático para prestar uma assistência diferenciada ao idoso em questão. Onde o envelhecimento, cada vez mais, está sendo uma realidade demográfica nas sociedades atuais, havendo assim a necessidade do profissional buscar conhecimento para 0 aperfeiçoamento e melhorar o atendimento e a demanda, uma vez que esta é crescente ${ }^{4}$.

\section{Planejamento e assistência da saúde do idoso na Estratégia Saúde da Família (ESF)}

Quanto ao planejamento e assistência da saúde do idoso na ESF, é importante planejar ações voltadas para a terceira idade, pois o processo de trabalho nas ESF é caracterizado pelo trabalho interdisciplinar articulado entre equipe, através da valorização dos conhecimentos de cada membro que a compõe, tendo a integralidade do sujeito e a resolutividade como eixo através do acompanhamento e avaliação sistemática das ações implementadas, visando a readequação do processo de trabalho 4 . 
Os profissionais entrevistados não têm planejamento de ações voltadas à saúde do idoso, como podemos observar em seus relatos:

E2; E3: Não.

E1; E4: realizam algum planejamento, nem sempre conseguem aplicá-lo.

E5: Prioridade no atendimento médico, e participação em alguns grupos: HAS, saúde mental.

O profissional enfermeiro apresenta dificuldade em realizar planejamento voltado a ações de enfermagem na promoção e prevenção do envelhecimento saudável. Entende-se que planejar é necessário, pois implica em ações em saúde que incorpore a identificação, a avaliação e o tratamento com perfis mórbidos e funcionais variados, que possam ser aplicados nas variadas modalidades assistenciais. Essas ações podem ser organizadas em níveis crescentes de complexidade e apropriadas para selecionar subgrupos dos indivíduos, com intuito de descrever características de risco, devem prosperar com distinção dentro da estrutura de atenção 4 .

Considerando que todo planejamento implica em ações de saúde do idoso, pois quando questionados sobre a saúde do idoso nas ESFs os entrevistados colocaram sobre a importância de planejar as ações, visto nas falas a seguir:

E1: [...] quando planejamos nossas ações e chamamos os idosos, geralmente eles comparecem e bem antes do horário marcado, ou quando não podem comparecer telefonam antecipadamente ou comparecem um dia antes justificando a ausência... Acredito que quanto mais ações criarmos mais o idoso irá comparecer, mas nem tudo que a gente orienta eles assimilam, por isso temos que ter um tom de voz mais alto para conversar fazer eles repetir o que explicamos e saber escutar 0 ponto de vista deles.

E2: Formações de profissionais devidamente preparados e qualificados para assistir a população idosa.

E3: Apesar das dificuldades, os nossos idosos estão de saúde e atendimentos bons, pois tentamos acolher da melhor maneira visando o seu bem estar.

E4: A ESF possui um número significativo de idosos, e apesar 
de não oferecermos ações específicas para esse grupo, procuramos estar sempre orientando o ACS para passar informações e trazer casos precisam de um maior cuidado e atenção da equipe.

E5: Toda a equipe precisa manter calma e paciência para lidar com os idosos, precisamos trazer eles para participação de atividades para podermos acompanhá-los mais de perto, e eles precisam ter um cuidado diferenciado quanto aos atendimentos/prioridades na sua idade.

Com base nos relatos mencionados, observa-se que os serviços possuem alguns objetivos que contemplam as demandas dos idosos, porém, não fica claro que haja um planejamento voltado para esta população, em especial ao envelhecimento e ao idoso. Espera-se que haja maior integração e uma aliança entre a equipe de saúde e o planejamento da assistência, com proposições direcionadas as ações e desenvolvendo de estratégias para o cuidado em fases específicas da vida do ser humano.

A falta de planejamento implica diretamente na necessidade de humanizar o cuidado, a assistência e a relação com o usuário do serviço de saúde. O SUS instituiu uma política pública de saúde que, apesar dos avanços, enfrenta ainda fragmentação do processo de trabalho e das relações entre os diferentes profissionais ${ }^{1}$.

Perante esse panorama, justifica-se a reflexão sobre a humanização na assistência à saúde, considerando a valorização dos diferentes sujeitos implicados nesse processo, tornando-se necessário repensar as políticas e práticas de assistência ao idoso para que o cuidado especial ao idoso seja realizado de forma humanizada. Visto ser um indivíduo que requer um atendimento e cuidado diferenciado, por ser uma idade também diferenciada ${ }^{6}$.

\section{CONCLUSÃO}

A população brasileira vem passando por mudanças no processo de envelhecimento. A redução significativa na taxa de fecundidade e o aumento da longevidade vêm trazendo desafio ao setor da saúde, com isso vem modificando o perfil epidemiológico do país. Diante desse processo percebe-se a necessidade 
emergente de investimentos na saúde, direcionadas a ações que promovam o envelhecer com qualidade de vida, ofertando à população condições e políticas de saúde com estratégias que possibilitem um envelhecer ativo, com dignidade e autonomia.

Diante deste novo cenário, a saúde pública precisa se "encorajar" e engajarse em políticas públicas de prevenção/promoção de saúde, tendo em vista que as pessoas estão vivendo por mais tempo, e com isso necessitam de uma saúde com mais qualidade e acesso aos serviços.

Com isso, entendemos que a hora de planejar e agir é agora, fazendo-se necessárias medidas para ajudar pessoas mais velhas a se manterem saudáveis e ativas e com qualidade de vida, sendo que isso é uma necessidade e um direito. As políticas públicas devem ser baseadas nos direitos, necessidades, preferências e habilidades, estabelecendo coerência entre as experiências de vida e entendendo que isso influencia na maneira e na forma como envelhecemos, pois uma vida mais longa deve ser acompanhada de oportunidades contínuas de saúde, proporcionando políticas que promovam saúde, autonomia e independência, incentivando as relações sociais.

Durante todo o percurso da pesquisa, evidenciou-se que os Enfermeiros das Estratégias Saúde da Família possuem dificuldades e grandes desafios no processo de trabalho, pois sendo a Atenção Primária em Saúde ordenadora do cuidado, percebe-se em suas falas que a falta de investimento de recursos humanos e gestão nos serviços dificulta o processo de trabalho. Por mais vontade que os profissionais tenham para trabalhar e desenvolver ações acerca do cuidado humanizado e que contribuam para um envelhecimento saudável e com qualidade de vida, deparam-se com dificuldades em prestar o atendimento, talvez pela sobrecarga de atividades, dividindo-se entre prestar assistência e administrar o serviço, dificultando assim à realização das ações de promoção e prevenção à saúde.

Envolver o idoso nas ações em prol do seu cuidado e valorizar seu conhecimento e sabedoria adquiridos durante a vida é uma forma de qualificar a assistência para esta idade. Há a necessidade de compreender o idoso e interagir com ele para que haja resolutividade nas ações realizadas pela equipe de saúde. Para isso, as ações precisam ser planejadas e organizadas a partir das necessidades 
Artigo Original

Atenção à Saúde

relatadas pelo próprio idoso, para que as mesmas apresentem melhores resultados no processo de cuidar e ser cuidado.

Conhecer as ações do enfermeiro nas Estratégias Saúde da Família quanto à promoção do envelhecimento saudável, nos reflete o quanto é importante para o desenvolvimento de ações para com o cuidado nesta idade e para a busca constante do aperfeiçoamento profissional.

Enfim, o planejamento da assistência para o enfermeiro é a consulta de enfermagem, instrumento primordial no seu processo de trabalho, permitindo planejamento da assistência diretamente com o paciente, proporcionado participação efetiva no processo de saúde.

Portanto, observamos que esse profissional tem dificuldade de realizar muitas das ações específicas da faixa etária, cabendo a ele o interesse por um aprendizado contínuo, a fim de alicerçar e fortalecer o conhecimento teórico-prático sobre os cuidados propostos ao idoso. Salientando a importância da capacitação do profissional para o reconhecimento das necessidades de saúde da pessoa idosa, respeitando suas limitações e potencialidades. Onde, mudanças de conceitos e paradigmas são necessários para que se tenha uma produção de saúde que contemple a pessoa idosa em todas suas dimensões, uma vez que se considera um grupo etário com características específicas e deve ser fortalecida pelos profissionais e serviços de saúde que prestam assistência.

\section{REFERÊNCIAS}

1. Carneiro IAF, Campino AC, Leite F, Rodrigues CG, Santos GM, Silva AR. Envelhecimento populacional e os desafios para o sistema de saúde brasileiro [Internet]. São Paulo: Instituto de Estudos de Saúde Suplementar; 2013.

2. Brasil. Ministério da Saúde. Secretaria de atenção á Saúde. Departamento de Atenção Básica no 19. Envelhecimento e Saúde da Pessoa Idosa. Cadernos de Atenção Básica. Brasília: Ministério da Saúde, 2006.

3. Minayo MCS. Pesquisa social: teoria, método e criatividade. Rio de Janeiro: Vozes; 2009.

4. Lenardt MH, Carneiro NHK, Albino J, Willig MH. Quality of life of frail elderly users of the primary care. Acta paul enferm 2014;27(5):399-404. 


\section{Atenção à Saúde}

5. Política Nacional de Atenção Básica [Internet]. Ministério da Saúde. 2012 [cited 10 mar. 2015]. Available from: http://www.dab.saúde.gov.br/portaldab/pnab.php.

6. Brasil. Ministério da Saúde. Portaria GM o․ 2488, de 21 de outubro de 2011. Aprova a Política Nacional de Atenção Básica, estabelecendo a revisão de diretrizes e normas para a organização da Atenção Básica, para a Estratégia Saúde da Família (ESF) eo Programa de Agentes Comunitários de Saúde (PACS). Brasília: Ministério da Saúde, 2011.

7. Oliveira JCA, Tavares DMdS. Atenção ao idoso na estratégia de Saúde da Família: atuação do enfermeiro. Rev esc enferm. 2009;44(3):774-81.

8. Santos AA, Monteiro EK, Póvoas FT, Lima LP, Silva FC. O papel do enfermeiro na promoção do envelhecimento saudável. Rev esp para a saúde. 2014;15(2):21-8.

9. Silvestre JA, Costa Neto MM. Abordagem do idoso em programas de saúde da família. Cad saúde pública. 2003;19(3):839-47.

10. Ferreira FPC, Bansi LO, Paschoal SMP. Serviços de atenção ao idoso e estratégias de cuidado domiciliares e institucionais. Rev bras geriatr gerontol. 2014;17(4):911-26.

11. Leopardi MT, Beck CLC, Nietsche EA, Gonzales RMB. Metodologia da pesquisa na saúde. Rio Grande do Sul: Pallotti; 2001.

12. Minayo MCS. O envelhecimento da população brasileira e os desafios para o setor saúde. Cad saúde pública. 2012;28(2):208-10.

13. Flesch LD, Araujo TCCF. Alta hospitalar de pacientes idosos: necessidades e desafios do cuidado contínuo. Estud psicol. 2014;19(3):227-36.

14. Brasil. Ministério da Saúde. Secretaria de Atenção à Saúde. Departamento de Atenção Básica. Cadernos de Atenção Básica. Brasília: Ministério da Saúde, 2013.

15. Januário IS, Magalhães LD, Fausto AK. Ações extensionistas voltadas para a promoção do envelhecimento saudável no município de Cuité-PB: Um relato de experiência. Rev UninCor. 2014;12(2):939-46.

16. Neri AL. Gerontologia. 4 ed. Campinas: Alínea; 2014.

17. Netto MP. Gerontologia: a velhice e o envelhecimento em visão globalizada. São Paulo: Atheneu; 2000. 